\title{
EXISTENCE CONDITIONS FOR A CLASS OF MODULAR SUBGROUPS OF GENUS ZERO
}

\author{
JOACHIM A. HEMPEL
}

Every subgroup of finite index of the modular group $P S L(2, \mathbb{Z})$ has a signature consisting of conjugacy-invariant integer parameters satisfying certain conditions. In the case of genus zero, these parameters also constitute a prescription for the degree and the orders of the poles of a rational function $F$ with the property:

$$
F^{\prime}(z)=0 \Longrightarrow \text { either } F(z)=F^{\prime \prime}(z)=0, F^{\prime \prime \prime}(z) \neq 0, \text { or } F(z)=1, F^{\prime \prime}(z) \neq 0 .
$$

Functions correspond to subgroups, and we use this to establish necessary and sufficient conditions for existence of subgroups with a certain subclass of allowable signatures.

\section{Preliminaries}

We identify the classical modular group $P S L_{2}(\mathbb{Z})$ with the group of self-mappings of the upper half plane $\mathcal{H}$, of the form $\tau \rightarrow(a \tau+b) /(c \tau+d)$ where $a, b, c, d$ are integers such that $a d-b c=1$. It is well known that the field of functions automorphic with respect to $P S L_{2}(\mathbb{Z})$ is the field of rational functions of just one such function $J$, analytic in $\mathcal{H}$, having multiplicity two at all points equivalent to $i$, multiplicity three at all points equivalent to $\rho$, and normalised by $J(i)=0, J(\rho)=1$, where $\rho=(1+i \sqrt{3}) / 2$.

Suppose now that $\Gamma$ is a subgroup of finite index of $P S L_{2}(\mathbb{Z})$, and of genus zero. Then again there exists a main function $\lambda$, referred to in German as the "Hauptmodul", such that the field of functions automorphic with respect to $\Gamma$ coincides with the rational functions of $\lambda$. Since $J$ is such a function, it follows that $J=F \circ \lambda$, where $F$ is a rational function. Since we are free to replace $\lambda$ by $M^{-1} \circ \lambda$, where $M$ is a Möbius transformation, we are also free to replace $F$ by $F \circ M$. In the sequel we shall call such a rational function, determined up to composition with $M$, the $J$-defining function for the genus zero subgroup.

In [3], the author described functions $\lambda$ in the case of no torsion, but in the general setting of Fuchsian groups. The present paper may be read for its results on rational Received 11th June, 2002.

Copyright Clearance Centre, Inc. Serial-fee code: 0004-9727/02 \$A2.00+0.00. 
functions with certain prescribed multiplicities, without any reference to modular subgroups.

We consider rational functions $F$ with the following properties

1. All points of multiplicity three are either zeros or poles of $F$,

2. All points of multiplicity two are either zeros of $F-1$ or poles of $F$,

3. All other multiple points of $F$ are poles of $F$.

These conditions can be stated in terms of the successive derivatives of $F$, for the case $z_{0} \neq \infty$ : If $F^{\prime}\left(z_{0}\right)=0$, then either $F\left(z_{0}\right)=F^{\prime \prime}\left(z_{0}\right)=0$ and $F^{\prime \prime \prime}\left(z_{0}\right) \neq 0$, or $F\left(z_{0}\right)=1$ and $F^{\prime \prime}\left(z_{0}\right) \neq 0$.

The conditions in the case $z_{0}=\infty$ are most easily stated in terms of the Laurent series at infinity. Suppose $F$ is finite at infinity and the Laurent series there is

$$
F(z)=\sum_{k=-\infty}^{0} a_{k} z^{k} .
$$

Then if $a_{-1}=0$ we have either $a_{0}=a_{-2}=0$ and $a_{-3} \neq 0$, or $a_{0}=1$ and $a_{-2} \neq 0$.

Taking advantage of the invariance of these conditions under Möbius transformations of the $z$-sphere, we can place a pole at infinity, and we do so throughout this paper. There is then a positive integer $\mu_{N}$ such that the Laurent series at infinity is

$$
F(z)=\sum_{k=-\infty}^{\mu_{N}} a_{k} z^{k},
$$

and interest attaches to the orders of the other poles. From the above conditions it is apparent that there exist polynomials $E_{2}, E_{3}, P, Q$ and $R$, which we assume to be monic, and a constant $c$ such that

$$
F(z)=\frac{P(z)^{3} E_{3}(z)}{c Q(z)},
$$

and

$$
F(z)-1=\frac{R(z)^{2} E_{2}(z)}{c Q(z)},
$$

where the product polynomial $E_{2} E_{3} P R$ has no zero of multiplicity greater than one, and shares no zero with $Q$.

There are many such sets of polynomials. A well known example is that corresponding to the expression for $J$ in terms of the standard Hauptmodul for the subgroup $\Gamma(2)$ :

$$
\begin{aligned}
& P(z)=z^{2}-z+1 \\
& R(z)=z^{3}-\frac{3}{2} z^{2}-\frac{3}{2} z+1
\end{aligned}
$$




$$
\begin{aligned}
Q(z) & =z^{2}(z-1)^{2}, \\
E_{3}(z) & =E_{2}(z)=1 \\
c & =\frac{27}{4} .
\end{aligned}
$$

Suppose that the degrees of the various polynomials are denoted by the corresponding lower case letters. For $j=1 \ldots N$, we denote by $\mu_{j}$ the orders of the poles of $F$, which, for $1 \leqslant j \leqslant N-1$, are the multiplicities of the distinct zeros $p_{j}$ of $Q$. We thus have

$$
Q(z)=\prod_{j=1}^{N-1}\left(z-p_{j}\right)^{\mu_{j}}
$$

and from (1.1) and (1.2) it is clear that

$$
\mu_{N}=3 p+e_{3}-q=2 r+e_{2}-q
$$

We denote the degree of $F$ by $\mu=\sum_{j=1}^{N} \mu_{j}$. Then

$$
\mu=3 p+e_{3}=2 r+e_{2} \text {. }
$$

For the reader's guidance, we note that capital letters other than $F$ and $N$ will denote polynomials. In writing the various equalities involving polynomials and their derivatives we shall usually omit explicit reference to the argument $z$, thus making sums and products those of functions rather than numbers.

We introduce the polynomial $S$ by

$$
S(z)=\prod_{j=1}^{N-1}\left(z-p_{j}\right)
$$

The greatest common divisor of $Q$ and its derivative $Q^{\prime}$ is the polynomial $Q / S$. We also introduce the polynomial $V$ by setting

$$
V=\frac{Q^{\prime} S}{Q}
$$

From (1.1) and (1.2) we have the following two formulae for the derivative of $F$

$$
F^{\prime}=\frac{P^{2}}{c Q S}\left(3 E_{3} P^{\prime} S+E_{3}^{\prime} P S-E_{3} P V\right),
$$

and

$$
F^{\prime}=\frac{R}{c Q S}\left(2 E_{2} R^{\prime} S+E_{2}^{\prime} R S-E_{2} R V\right)
$$


Since $P$ and $R$ have no common factor and between them account for all the zeros of $F^{\prime}$, there exists a constant $k$ such that

$$
\begin{aligned}
k P^{2} & =2 E_{2} R^{\prime} S+E_{2}^{\prime} R S-E_{2} R V \\
k R & =3 E_{3} P^{\prime} S+E_{3}^{\prime} P S-E_{3} P V .
\end{aligned}
$$

The following lemma is simply the Riemann-Hurwitz relation stated for the function $F$, and we give the short direct proof.

LEMma 1. The number $N$ of distinct poles of $F$, the degrees $e_{2}, e_{3}$ of the polynomials $E_{2}, E_{3}$, and the degree $\mu$ of $F$ are related by

$$
\mu=6(N-2)+3 e_{2}+4 e_{3} .
$$

Proof: From the equalities just displayed it follows that

$$
F^{\prime}=\frac{k P^{2} R}{c Q S}
$$

From this we see that, at infinity

$$
F^{\prime}(z)=\frac{k}{c} z^{2 p+r-q-N+1}+\ldots
$$

On the other hand from (1.1) we have

$$
F(z)=\frac{1}{c} z^{\mu_{N}}+\ldots
$$

and so

$$
F^{\prime}(z)=\frac{\mu_{N}}{c} z^{\mu_{N}-1}+\ldots
$$

Comparing these expressions we see that $k=\mu_{N}$ and that

$$
\mu_{N}-1=2 p+r-q-N+1 .
$$

From (1.4) it follows that

$$
\mu_{N}-1=\frac{2}{3}\left(\mu-e_{3}\right)+\frac{1}{2}\left(\mu-e_{2}\right)-\mu+\mu_{N}-N+1,
$$

and so

$$
\frac{\mu}{6}=N-2+\frac{2}{3} e_{3}+\frac{1}{2} e_{2}
$$

which was to be proved.

We note that the numbers $\mu, e_{3}, e_{2}$ and $N$ are Möbius invariant and the lemma holds regardless of whether or not there is a pole at infinity. As a bonus in the case of our normalisation we obtain that $k=\mu_{N}$, and we note for future reference the formulae

$$
\mu_{N} P^{2}=2 E_{2} R^{\prime} S+E_{2}^{\prime} R S-E_{2} R V,
$$




$$
\begin{aligned}
\mu_{N} R & =3 E_{3} P^{\prime} S+E_{3}^{\prime} P S-E_{3} P V, \\
F^{\prime} & =\frac{\mu_{N} P^{2} R}{c Q S} .
\end{aligned}
$$

The significance for the subgroup $\Gamma$ of the various parameters is as follows. Referring to a connected fundamental domain for $\Gamma$, the numbers $e_{j}$ for $j=2,3$ are the numbers of inequivalent fixed point vertices of order $j, N$ is the number of inequivalent cusps, and $\mu$ is the index of $\Gamma$ in the modular group. The orders $\mu_{k}$ of the poles of $F$ are the cusp widths, or fan widths, of the inequivalent cusps. For further information we refer the reader to Gunning [2].

In Section 2 we state and prove our main result, leaving till Section 3 the proof of a key lemma.

\section{The Main Result}

We now adopt a different convention regarding the labelling of the cusp widths, or equivalently, the orders of the poles of $F$. Let $\mu_{1} \ldots \mu_{K}$ be the distinct cusp widths that occur in $\Gamma$ and let $\nu_{k}$ be the frequency of $\mu_{k}$, that is, the number of inequivalent cusps with width $\mu_{k}$. Then clearly $\sum_{k=1}^{K} \nu_{k}=N$, and $\sum_{k=1}^{K} \nu_{k} \mu_{k}=\mu$.

An array of integers $\left(\mu ; N, e_{2}, e_{3}, K, \mu_{k}, \nu_{k}\right)$, with $e_{2}, e_{3} \geqslant 0$, and $\mu, N, K, \mu_{k}, \nu_{k} \geqslant 1$ and satisfying the Riemann-Hurwitz relation

$$
\sum_{k=1}^{K} \nu_{k} \mu_{k}=\mu=6(N-2)+3 e_{2}+4 e_{3},
$$

will be referred to as an allowable signature. Not every allowable signature occurs as the signature of some modular subgroup. We shall say that such signatures are not realised.

Definition 2: We say that $\Gamma$ is of low torsion and high frequency, if for some $k$,

$$
\mu_{k} \geqslant 2 \text {, and } \nu_{k} \geqslant N-2+e_{2}+e_{3} .
$$

It is clear that, for a low torsion and high frequency subgroup, $1 \leqslant k \leqslant K \leqslant 3$, and $e_{2}+e_{3} \leqslant 2$.

To state our main Theorem we first define the rational functions $F_{2}, F_{3}, F_{4}, F_{5}$, by

$$
\begin{aligned}
& F_{2}(z)=\frac{4\left(z^{2}-z+1\right)^{3}}{27 z^{2}(z-1)^{2}}, \\
& F_{3}(z)=\frac{\left(z^{4}+4 z^{3}+6 z^{2}+12 z+9\right)^{3}}{64 z^{3}\left(z^{2}+3 z+3\right)^{3}}, \\
& F_{4}(z)=\frac{\left(z^{2}+14 z+1\right)^{3}}{108 z(z-1)^{4}} \\
& F_{5}(z)=\frac{\left(z^{4}+228 z^{3}+494 z^{2}-228 z+1\right)^{3}}{1728 z\left(z^{2}-11 z-1\right)^{5}} .
\end{aligned}
$$


THEOREM 3. The only low torsion and high frequency subgroups of genus zero that exist are those with the following signatures and $J$-defining functions $F$ :

(i) $\left(\mu ; N, e_{2}, e_{3}, K, \mu_{k}, \nu_{k}\right)=\left(3 ; 2,1,0,2, \mu_{1}=2, \mu_{2}=1, \nu_{1}=1, \nu_{2}=1\right)$,

$$
F(z)=\frac{(z+2)^{3}}{27 z}
$$

(ii) $\left(\mu ; N, e_{2}, e_{3}, K, \mu_{k}, \nu_{k}\right)=\left(4 ; 2,0,1,2, \mu_{1}=3, \mu_{2}=1, \nu_{1}=1, \nu_{2}=1\right)$,

$$
F(z)=\frac{(z+1)^{3}(z+9)}{64 z}
$$

(iii) For $\mu_{1}=2,3,4,5$,

$$
\begin{aligned}
& \left(\mu ; N, e_{2}, e_{3}, K, \mu_{k}, \nu_{k}\right)=\left(\frac{12 \mu_{1}}{6-\mu_{1}} ; \frac{12}{6-\mu_{1}}, 0,0,1, \mu_{1}, \nu_{1}=\frac{12}{6-\mu_{1}}\right), \\
& F(z)=F_{\mu_{1}}\left(z^{N-2}\right) \text { for } \mu_{1}=2,4, \text { and } F(z)=F_{\mu_{1}}\left(z^{(N-2) / 2}\right) \text { for } \mu_{1}=3,5 .
\end{aligned}
$$

(iv) For $\mu_{1}=2,3,4,5, N \geqslant 3, \mu_{1} N$ even, $\mu_{1} N \neq 6(N-2)$,

$$
\left(\mu ; N, e_{2}, e_{3}, K, \mu_{k}, \nu_{k}\right)=\left(6(N-2) ; N, 0,0,2, \mu_{1}, \mu_{2}=\frac{\mu}{12}\left(6-\mu_{1}\right), \nu_{1}=N-2, \nu_{2}=2\right) \text {, }
$$$$
F(z)=F_{\mu_{1}}\left(z^{N-2}\right) \text { for } \mu_{1}=2,4 \text {, and } F(z)=F_{\mu_{1}}\left(z^{(N-2) / 2}\right) \text { for } \mu_{1}=3,5 \text {. }
$$

Note that case (iii) can be subsumed in case (iv) by allowing $\mu_{1}=\mu_{2}$.

It follows that there are allowable signatures with arbitrarily large values of $\mu$, which are not realised. This answers in the negative a question posed by Millington [5, p. 356].

When $N=4$ and $e_{2}=e_{3}=0$ there are fifteen allowable signatures, six of which are realised. Of these six, three are low torsion and high frequency. Of the nine non-realised signatures six correspond to low torsion and high frequency cases excluded by the above Theorem. When $N=5$ and $e_{2}=e_{3}=0$ there are fifty eight allowable signatures, twenty six of which are realised. Of these twenty six, two are low torsion and high frequency. Of the thirty two non-realised signatures twelve correspond to low torsion and high frequency cases excluded by the above Theorem. A broader aim motivating the present work is to account for non-realisation in all cases.

Much recent work has been devoted to the identification of congruence subgroups, see Hsu [4] and the references quoted therein. The present work makes no distinction between congruence and noncongruence subgroups. We remark that, in the torsionfree genus zero case, all subgroups are congruence when $N=4$, and no subgroups are congruence when $N=5$. For details see Sebbar [6].

We defer to Section 3 the proof of the following key lemma.

LEMMA 4. Suppose that $\Gamma$ is a low torsion and high frequency subgroup, and that $\mu_{1}$ is the frequent cusp width of the definition. If there exists $\mu_{k} \neq \mu_{1}$ then $12 \mu_{k}$ $=\mu\left(6-\mu_{1}\right)$. Otherwise $12 \mu_{1}=\mu\left(6-\mu_{1}\right)$. 
Proof of Theorem 3: Suppose first that $\mu_{1}$ is the only cusp width that occurs. Then $\mu=N \mu_{1}$, by the first equality in (2.1), and then Lemma 4 implies that

$$
12=N\left(6-\mu_{1}\right) .
$$

The only solutions in positive integers of this equation are $\mu_{1}=2,3,4,5$, with $N$ $=3,4,6,12$ respectively. Comparing the same equality with the right hand equality in (2.1), we see that $e_{2}=e_{3}=0$. This gives us case (iii) of the Theorem.

Suppose now that $\nu_{1}=N-1, \nu_{2}=1$. It follows that $e_{2}+e_{3} \leqslant 1$, and so either $e_{2}=1, e_{3}=0$, or $e_{2}=0, e_{3}=1$. In either case (2.1) can be written in the form

$$
\mu_{1}+\mu_{2}=\left(6-\mu_{1}\right)(N-2)+3 e_{2}+4 e_{3} .
$$

On the other hand Lemma 4 gives

$$
12 \mu_{2}=6\left(6-\mu_{1}\right)(N-2)+\left(6-\mu_{1}\right)\left(3 e_{2}+4 e_{3}\right) .
$$

From these we obtain

$$
6\left(\mu_{1}-\mu_{2}\right)=\mu_{1}\left(3 e_{2}+4 e_{3}\right),
$$

and we deduce cases (i) and (ii) of Theorem 3 if $e_{2}+e_{3}=1$. (If $e_{2}=e_{3}=0$ we have $\mu_{1}=\mu_{2}$, which gives case (iii) again).

Finally we suppose that $\nu_{1}=N-2$, and $\nu_{2}=2$. Then $e_{2}=e_{3}=0$, and (2.1) reduces to

$$
2 \mu_{2}=\left(6-\mu_{1}\right)(N-2),
$$

which is equivalent to the conclusion of Lemma 4 . The significance of Lemma 4 in this case lies in the exclusion of a distinct $\mu_{3}$. Thus we deduce case (iv) of Theorem 3.

The existence of $J$-defining functions $F$ in all cases of Theorem 3 is a matter of calculation of their respective formulae, which we leave as exercises for the interested reader. Alternatively the reader can simply verify that the given formulae satisfy the description of Section 1. This completes the proof of Theorem 3.

\section{Proof of Lemma 4}

This Section is a continuation of Section 1, where we established the formulae (1.68). In [1] Atkin and Swinnerton-Dyer had suggested taking derivatives in simplifying the task of finding the $J$-defining function by equating coefficients in the identity

$$
P(z)^{3} E_{3}(z)-R(z)^{2} E_{2}(z)-c Q(z)=0 .
$$

The basic idea is expressed in (1.8), namely, the zeros of $F^{\prime}$ are double zeros where $P=0$, and simple zeros where $R=0$. We develop this idea further. 
We do not apply the conditions of Lemma 4 till the very end, so the reader may regard this Section as presenting the groundwork for algorithms for finding $J$-defining functions in the general case of genus zero. In fact such algorithms were used for both finding the functions $F_{j}$ appearing in the Theorem 3 , and enumerating the genus zero cases with $N=4,5$ and 6 .

We anticipate the situation of Lemma 4 by setting the $\mu_{k}$ of that Lemma equal to $\mu_{K}$. Instead of (1.3), we now write,

$$
Q=\prod_{j=1}^{K} S_{j}^{\mu_{j}} \text { and } S=\prod_{j=1}^{K} S_{j}
$$

where the $S_{j}$ are monic polynomials whose distinct zeros are those zeros of $Q$ which are of order $\mu_{j}$. The sum of the degrees of the $S_{j}$ is $N-1$, and we note that $\operatorname{deg} S_{j}=\nu_{j}$ for $j<K$ and $\operatorname{deg} S_{K}=\nu_{K}-1$. Recalling that the last pole order $\mu_{K}$ is the order of the pole of $F$ at infinity, we rewrite (1.6) and (1.7) as

$$
\begin{aligned}
\mu_{K} P^{2} & =2 E_{2} R^{\prime} S+E_{2}^{\prime} R S-E_{2} R V, \\
\mu_{K} R & =3 E_{3} P^{\prime} S+E_{3}^{\prime} P S-E_{3} P V .
\end{aligned}
$$

On formally eliminating $R$ between these identities we find that

$$
P\left(\mu_{K}^{2} P-E_{3} E_{2} V^{2}\right)=S\left(2 \mu_{K} E_{2} R^{\prime}-3 E_{3} E_{2} V P^{\prime}+3 E_{3} E_{2}^{\prime} S P^{\prime}-E_{3}^{\prime} E_{2} V P-E_{3} E_{2}^{\prime} V P+E_{3}^{\prime} E_{2}^{\prime} S P\right) \text {. }
$$

Since $P$ and $S$ are coprime we have a polynomial $U$ such that

$$
\mu_{K}^{2} P=E_{3} E_{2} V^{2}+S U
$$

and

$$
2 \mu_{K} E_{2} R^{\prime}=3 E_{3} E_{2} V P^{\prime}+U P-3 E_{3} E_{2}^{\prime} S P^{\prime}+E_{3}^{\prime} E_{2} V P+E_{3} E_{2}^{\prime} V P-E_{3}^{\prime} E_{2}^{\prime} S P .
$$

By examining the leading terms in (3.4) we see that $U$ has leading term $\mu\left(2 \mu_{K}-\mu\right) z^{N-3+e_{3}+e_{2}}$ when this is non-zero, and thus has degree at most $N-3+e_{3}+e_{2}$.

From (3.3) we obtain

$$
\mu_{K} R^{\prime}=3 E_{3} S P^{\prime \prime}+\left(3\left(E_{3} S\right)^{\prime}+E_{3}^{\prime} S-E_{3} V\right) P^{\prime}+\left(\left(E_{3}^{\prime} S\right)^{\prime}-\left(E_{3} V\right)^{\prime}\right) P .
$$

Substituting this in (3.5) we have, after some tidying,

$$
\begin{aligned}
& 6 E_{3} E_{2} S P^{\prime \prime}+\left(6 E_{3} E_{2} S^{\prime}-5 E_{3} E_{2} V+8 E_{3}^{\prime} E_{2} S+3 E_{3} E_{2}^{\prime} S\right) P^{\prime} \\
& +\left(-U-2 E_{3} E_{2} V^{\prime}+2 E_{3}^{\prime \prime} E_{2} S+2 E_{3}^{\prime} E_{2} S^{\prime}-3 E_{3}^{\prime} E_{2} V+E_{3}^{\prime} E_{2}^{\prime} S-E_{3} E_{2}^{\prime} V\right) P=0 .
\end{aligned}
$$

From (3.4) we see that

$$
\mu_{K}^{2} P^{\prime}=E_{3}^{\prime} E_{2} V^{2}+E_{3} E_{2}^{\prime} V^{2}+2 E_{3} E_{2} V V^{\prime}+S^{\prime} U+S U^{\prime} .
$$


We could differentiate again and substitute in the previous expression to eliminate $P$ and its derivatives, but refrain from doing so. Instead we examine what happens at points where $S=0$. On setting $S=0$ in (3.4) and (3.7), and substituting the resulting expressions for $P$ and $P^{\prime}$ in (3.6), we see that when $S=0$,

$$
\begin{aligned}
E_{3} E_{2}\left(6 S^{\prime}-5 V\right) & \left(E_{3}^{\prime} E_{2} V^{2}+E_{3} E_{2}^{\prime} V^{2}+2 E_{3} E_{2} V V^{\prime}+S^{\prime} U\right) \\
+ & \left(-U-2 E_{3} E_{2} V^{\prime}+2 E_{3}^{\prime} E_{2} S^{\prime}-3 E_{3}^{\prime} E_{2} V-E_{3} E_{2}^{\prime} V\right) E_{3} E_{2} V^{2}=0 .
\end{aligned}
$$

Since $E_{3} E_{2} \neq 0$ at points where $S=0$ we can divide by $E_{3} E_{2}$ and obtain, after a factorisation,

$$
\left(S^{\prime}-V\right)\left(12 E_{3} E_{2} V V^{\prime}+6 E_{3} E_{2}^{\prime} V^{2}+8 E_{3}^{\prime} E_{2} V^{2}+U V+6 S^{\prime} U\right)=0,
$$

at points at which $S=0$.

We now observe from the expressions (1.3) and (1.5) for $Q$ and $V$, that, if the order of $p_{j}$ is $\mu_{j}$, then

$$
V\left(p_{j}\right)=\mu_{j} S^{\prime}\left(p_{j}\right)
$$

For $\mu_{j}=1$, we have $S^{\prime}-V=0$ at such points, so (3.8) gives no information; but for $\mu_{j}>1$ it implies that $S_{j}$ divides $\left(12 E_{3} E_{2} V^{\prime}+6 E_{3} E_{2}^{\prime} V+8 E_{3}^{\prime} E_{2} V+U\right) \mu_{j}+6 U$.

The leading term in this expression is $\mu\left(12 \mu_{K}-\mu\left(6-\mu_{j}\right)\right) z^{N-3+e_{3}+e_{2}}$. It follows that if $12 \mu_{K} \neq \mu\left(6-\mu_{j}\right)$, and $\mu_{j} \geqslant 2$ then the degree of $S_{j}$, namely $\nu_{j}$, can not be larger than $N-3+e_{3}+e_{2}$. Conversely, if $\nu_{j} \geqslant N-2+e_{3}+e_{2}$, we have $12 \mu_{K}=\left(6-\mu_{j}\right) \mu$.

With $j=1$, this is Lemma 4.

\section{REFERENCES}

[1] A.O.L. Atkin and H.P.F. Swinnerton-Dyer, 'Modular forms on noncongruence subgroups', in Proc. Sympos. Pure Math. 19 (American Mathematical Society, Providence, R.I., 1971), pp. 1-25.

[2] R.C.Gunning, Lectures on modular forms, Annals of Math. Studies 48 (Princeton University Press, Princeton, 1962).

[3] J.A. Hempel, 'On the uniformization of the n-punctured sphere', Bull. London Math. Soc. 20 (1988), 97-115.

[4] T. Hsu, 'Identifying congruence subgroups of the modular group', Proc. Amer. Math. Soc. 124 (1996), 1351-1359.

[5] M.H. Millington, 'Subgroups of the classsical modular group', J. London Math Soc. 21 (1969), 351-357.

[6] A. Sebbar, 'Classification of torsion-free genus zero congruence subgroups', Proc. Amer. Math. Soc. 129 (2001), 2517-2527.

School of Mathematics and Statistics

University of Sydney

New South Wales 2006

Australia

e-mail: joachimh@maths.usyd.edu.au 